\begin{tabular}{|c|c|c|c|}
\hline DE & \multirow{3}{*}{$\begin{array}{l}\text { DE GRUYTER } \\
\text { OPEN }\end{array}$} & $\begin{array}{l}\text { HUNGARIAN JOURNAL OF } \\
\text { INDUSTRY AND CHEMISTRY }\end{array}$ & \multirow[t]{3}{*}{$\begin{array}{l}\text { IHUNGARLAN JOURNAII OF } \\
\text { INIDUSTIRY AND CIIEMISIRSY }\end{array}$} \\
\hline & & Vol. 44(2) pp. 113-120 (2016) & \\
\hline & & $\begin{array}{l}\text { hjic.mk.uni-pannon.hu } \\
\text { DOI: } 10.1515 / h j i c-2016-0014\end{array}$ & \\
\hline
\end{tabular}

\title{
COMPUTATIONAL STABILITY ANALYSIS OF LOTKA-VOLTERRA SYSTEMS
}

\author{
PÉTER POLCZ ${ }^{1, *}$ AND GÁBOR SZEDERKÉNYI ${ }^{1,2}$ \\ ${ }^{1}$ Faculty of Information Technology and Bionics, Pázmány Péter Catholic University, Práter u. 50/A, \\ Budapest, 1083, HUNGARY \\ 2 Systems and Control Lab, Institute for Computer Science and Control, Hungarian Academy of \\ Sciences, Práter u. 50/A., Budapest, 1083, HUNGARY
}

\begin{abstract}
This paper concerns the computational stability analysis of locally stable Lotka-Volterra (LV) systems by searching for appropriate Lyapunov functions in a general quadratic form composed of higher order monomial terms. The Lyapunov conditions are ensured through the solution of linear matrix inequalities. The stability region is estimated by determining the level set of the Lyapunov function within a suitable convex domain. The paper includes interesting computational results and discussion on the stability regions of higher $(3,4)$ dimensional LV models as well as on the monomial selection for constructing the Lyapunov functions. Finally, the stability region is estimated of an uncertain 2D LV system with an uncertain interior locally stable equilibrium point.
\end{abstract}

Keywords: nonlinear systems, Lotka-Volterra models, stability analysis, linear matrix inequalities, Lyapunov function

\section{Introduction}

Approximating the domain of attraction (DOA) is often a fundamental task in the analysis and control of nonlinear systems. The stability properties of dynamical systems are most often studied using Lyapunov functions. Therefore, extensive literature exists on the computational construction of Lyapunov functions [1].

Due to their advantageous properties and the availability of efficient numerical solvers, the use of linear matrix inequalities (LMI) and semi-definite programming (SDP) techniques has become popular in the field of system and control theory. Important results were announced $[2,3]$ in the context of linear uncertain systems, their stability analysis and control synthesis.

Recently, an optimization-based method for DOA estimation was published [4], where the authors use Finsler's lemma and affine parameter-dependent LMIs to compute rational Lyapunov functions for a wide class of locally asymptotically stable nonlinear systems. Based on these results an improved method was published $[5,6]$, where the transformation of the model to the form required for optimization is done automatically using the linear fraction transformation (LFT) and further automatic model simplification steps, which results in the dimension reduction of the optimization task. As the dimensions of the problem are

*Correspondence: ppolcz@gmail.com reduced, the method is capable of handling more complex models. For example, the DOA is successfully estimated for a 3-dimensional rational uncertain system [6] (bioreactor model with an applied proportional and integral substrate feedback law).

The dynamical descriptive power of LotkaVolterra systems is so extensive that LV models "have the status of canonical format" within the class of smooth nonlinear dynamical systems [7]. Besides modelling biological/ecological environments, they are widely used in other scientific fields like neural networks [8] or in economics, where the GoodwinLotka-Volterra models are applied for modelling the predator-prey mechanism of the technological substitution $[9,10]$. In order to model the correlation between the employment rate and the share of wages of the working population, the authors of Ref. [11] used a stochastic extension of the Goodwin model.

Some important results that make LV models even more attractive are the existing techniques used to represent a general nonlinear system as a multidimensional LV model [12]. The analysis of stability and behaviour of LV systems is extensive in the literature [13]. Plank has shown [14] that $N$ dimensional LV systems are Hamiltonian if they fulfil certain algebraic properties (Theorem 3.1 in Ref. [14]). He demonstrated that, when using an appropriate Poisson structure, one can obtain the Hamiltonian function of the system, which is a key object in determining the system's DOA. Furthermore, candidates of Hamiltonian function were defined in (Section IV in Ref. [14]). It is important to note that LV systems are 
kinetic in the sense that they can be formally described as chemical reaction networks with mass action kinetics.

In this paper, the local stability properties of LV models are analysed by applying the underlying method presented [6]. The stability region is estimated on a few locally stable 2-, 3-, and 4-dimensional benchmark LV systems. The DOA of an uncertain $2 \mathrm{D}$ system is also estimated by two concentric regions.

The main motivation of our work was to evaluate the applicability of the approach [4] on a general polynomial system class consisting of low-degree monomials, and to study the limits of the method as the number of dimensions of the state space increase.

\section{Background}

In this section, the basic notions and results on which our computational method is based are presented.

\subsection{System Class, Lyapunov Functions, and the Domain of Attraction.}

Our computational method can handle general nonlinear systems of the form

$$
\begin{gathered}
\dot{x}(t)=f(x(t), \delta(t)) \\
x(t) \in \mathbb{R}^{\mathrm{N}}, x_{0} \in \mathcal{X}, \delta(t) \in \mathcal{D}, \dot{\delta}(t) \in \widetilde{\mathcal{D}}
\end{gathered}
$$

where $\mathcal{X} \subset \mathbb{R}^{\mathrm{N}}$ and $\mathcal{D}, \breve{\mathcal{D}} \subset \mathbb{R}^{\mathrm{D}}$ are given polytopes, $x$ is the state vector function with its initial condition $x_{0}=$ $x(0)$, and $\delta$ is a smooth, bounded vector function of uncertain parameters with a bounded time derivative. The applied method is presented in detail elsewhere [15]. From now on, the time arguments of $x$ and $\delta$ will be suppressed as is commonly done in the literature. It is assumed that function $f: \mathbb{R}^{\mathrm{N}} \times \mathcal{D} \mapsto \mathbb{R}^{\mathrm{N}}$ of $E q .(1)$ is a well-defined smooth rational mapping, with the property $f(0, \delta)=0$ for all $\delta \in \mathcal{D}$. Additionally, it is assumed that $x^{*}=0 \in \mathbb{R}^{\mathrm{N}}$ is a locally asymptotically stable equilibrium point of $E q$.(1) for all $\delta \in \mathcal{D}$. The set of all initial conditions, from which the solutions converge to $x^{*}$, is called the domain of attraction (DOA). Furthermore, it is assumed that function $f(x, \delta)$ can be written in a so-called quasi-LPV form $f(x, \delta)=$ function $A(x, \delta) x$

The aim is to identify an appropriate rational Lyapunov function $V(x, \delta)$, which satisfies the following conditions:

$$
\begin{aligned}
& v_{1}(x) \leq V(x, \delta) \leq u_{\mathrm{x}}(x), \forall(x, \delta) \in X \times \mathcal{D} \\
& \dot{V}(x, \delta, \dot{\delta}) \leq-v_{\mathrm{d}}(x) \forall(x, \delta, \dot{\delta}) \in X \times \mathcal{D} \times \overline{\mathcal{D}}
\end{aligned}
$$

where $v_{1}, u_{\mathrm{x}}, v_{\mathrm{d}}$ are continuous positive functions on $X$. Due to conditions of Eq.(2) any closed level set of the Lyapunov function contained entirely in $X$ bounds an invariant region of the state space. Our objective is to find a Lyapunov function, which fulfils the conditions of Eq.(2) and to determine its maximal invariant level set completely inside $\mathcal{X}$.

\subsection{Dynamical System Representation}

A Lyapunov function can be computed [4] in the form shown in Eq.(3).

$$
V(x, \delta)=\pi_{\mathrm{b}}^{\mathrm{T}}(x, \delta) P \pi_{\mathrm{b}}(x, \delta), \pi_{\mathrm{b}}=\left[\begin{array}{l}
x \\
\pi
\end{array}\right]
$$

where $P \in \mathbb{R}^{\mathrm{m} \times \mathrm{m}}$ is a constant symmetric matrix, not necessarily positive definite, and $\pi: \mathbb{R}^{\mathrm{N}} \times \mathcal{D} \mapsto \mathbb{R}^{\mathrm{p}}$ is mapping, in which each element is a monomial in $(x, \delta)$, or a smooth rational function with a monomial numerator. The arguments of $\pi$ and $\pi_{b}$ will be suppressed below.

Applications of the linear fractional transformation (LFT) and further algebraic steps have been proposed $[5,6]$ to transform the system equation $\dot{x}=f(x, \delta)=$ $A(x, \delta) x$ into the desired differential-algebraic representation that was introduced in the same references:

$$
\begin{aligned}
& \dot{x}=A(x, \delta) x=A x+B \pi \quad x_{0} \in \mathcal{X} \\
& 0=\mathcal{N}_{\pi_{\mathrm{b}}}(x, \delta) \pi_{\mathrm{b}} \quad \delta \in \mathcal{D}, \dot{\delta} \in \check{\mathcal{D}}
\end{aligned}
$$

where $A \in \mathbb{R}^{\mathrm{n} \times \mathrm{n}}$ and $B \in \mathbb{R}^{\mathrm{n} \times \mathrm{p}}$ are constant matrices, and $\mathcal{N}_{\pi_{\mathrm{b}}}(x, \delta) \in \mathbb{R}^{\mathrm{q} \times \mathrm{m}}$ is an affine matrix function in ( $x$, $\delta)$ also known as an "annihilator". These transformations also result in the dimension reduction of the optimization problem compared to the results presented in Ref. [4]. The representation in Eq.(4) separates the linear part of the system $(x)$ from its nonlinear part $(\pi)$.

\section{The SDP Problem for Estimating the DOA}

In this section, how to construct the semi-definite optimization task (SDP) is presented, which will uniquely characterize an appropriate Lyapunov function and its maximal invariant level set $\varepsilon_{\alpha}$ as an estimate of the true domain of attraction. It is assumed that polytopes $\mathcal{X}, \mathcal{Y}$, and $\mathcal{D}$ are already given.

Using the model representation of Eq.(4) and a Lyapunov function candidate of the form in Eq.(3), sufficient LMIs for the Lyapunov conditions in Eq.(2) can be formulated. According to Finsler's lemma (Lemma 2.1 in Ref. [4]), if real-valued matrices $L_{\mathrm{b}} \in \mathbb{R}^{\mathrm{m} \times \mathrm{q}}$ and $L_{\mathrm{a}} \in \mathbb{R}^{\left(\mathrm{n}+2 \mathrm{p}+\mathrm{n}^{2}+\mathrm{np}\right) \times\left(2 \mathrm{q}+\mathrm{n}^{2}+\mathrm{nq}\right)}$ exist

$$
\begin{gathered}
\forall(x, \delta) \in \vartheta(x \times \mathcal{D}): \\
P+L_{\mathrm{b}} \mathcal{N}_{\pi_{\mathrm{b}}}(x, \delta)+\mathcal{N}_{\pi_{\mathrm{b}}^{\mathrm{b}}}^{\mathrm{T}}(x, \delta) L_{\mathrm{b}}^{\mathrm{T}}>0 \\
\forall(x, \delta, \dot{\delta}) \in \vartheta(x \times \mathcal{D} \times \widetilde{\mathcal{D}}): \\
P_{\mathrm{a}}+P_{\mathrm{a}}^{\mathrm{T}}+L_{\mathrm{a}} \mathcal{N}_{\pi_{\mathrm{a}}}(x, \delta, \dot{\delta})+\mathcal{N}_{\pi_{\mathrm{a}}}^{\mathrm{T}}(x, \delta, \dot{\delta})<0
\end{gathered}
$$

then the conditions of Eq.(2) are satisfied for every $(x, \delta, \dot{\delta}) \in \mathcal{X} \times \mathcal{D} \times \widetilde{\mathcal{D}}$. Variables $P_{\mathrm{a}}$ and $\mathcal{N}_{\pi_{\mathrm{a}}}(x, \delta, \dot{\delta})$ are 


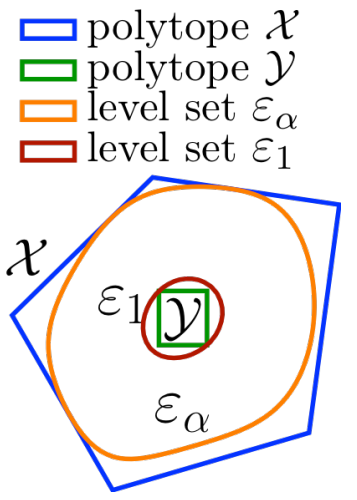

Figure 1. Illustration of the conditions regarding $X$ and $\mathcal{Y}$. Inside polytope $\mathcal{X}$ of the Lyapunov conditions in Eq.(2) are required. The $\alpha$-level set $\varepsilon_{\alpha}$ of the Lyapunov function should lie inside polytope $\mathcal{X}$ (hence it is invariant). The 1-level set $\varepsilon_{1}$ should be around polytope $\mathcal{Y}$. This condition ensures that the problem has a bounded solution.

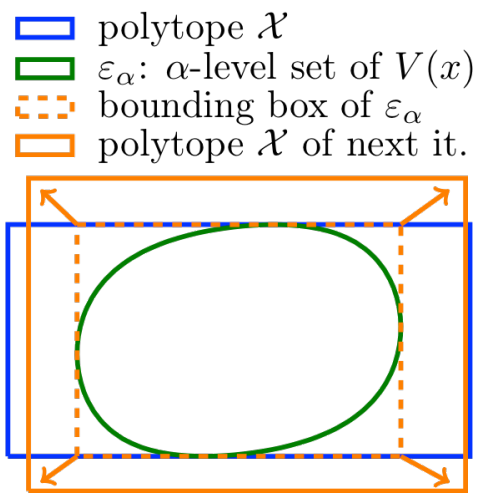

Figure 2. Polytope $\mathcal{X}$ is evaluated through iterations considering the bounding box of the obtained $\alpha$-level set.

defined in Eqs.(3.16) and (3.22) of Ref. [15], respectively. $\vartheta(\cdot)$ denotes the corner points (vertices) of a given polytope.

Since the Lyapunov conditions are satisfied within $\mathcal{X}$, an attempt is made to identify the maximal $\alpha$-level set

$$
\varepsilon_{\alpha}=\{x \in \mathcal{X} \mid V(x)=\alpha, 1 \leq \alpha\}
$$

which lies inside $\mathcal{X}$. That level set will be invariant, in the sense that every trajectory entering this region will never leave it.

$\alpha \geq 1$ is defined, as a free variable of the optimization task, and $\varepsilon_{\alpha}$ is constrained inside $\mathcal{X}$. One can observe that by maximizing $\alpha$ an unbounded feasible solution is obtained, as the function $V(x, \delta)$ can be scaled arbitrarily. Therefore, as Fig.1 illustrates, an auxiliary polytope $\mathcal{Y}$ is defined around the locally stable origin inside $\chi$, constraining the 1-level set $\varepsilon_{1}=\{x \in$ $x \mid V(x)=1\}$ to be around $\mathcal{Y}$.

According to Finsler's lemma, for every $k=\overline{1, M_{X}}$ and $l=\overline{1, M_{y}}$, if real-valued matrices $L_{\mathrm{C}_{\mathrm{k}}}, \bar{L}_{\mathrm{C}_{\mathrm{l}}} \in \mathbb{R}^{\mathrm{m} \times \mathrm{q}}$, $M_{\mathrm{C}_{\mathrm{k}}}, \bar{M}_{\mathrm{C}_{\mathrm{l}}} \in \mathbb{R}^{(\mathrm{m}+1) \times \mathrm{n}}$ exit
Table 1. Number and dimensions of parameter independent LMIs of the optimization task.

\begin{tabular}{lll}
\hline & Dimension of the LMIs & \multicolumn{1}{c}{ No. of LMIs } \\
\hline Eq.(5) & $m$ & $M_{X} \cdot M_{\mathcal{D}}$ \\
Eq.(6) & $n+2 p+n^{2}+n p$ & $M_{X} \cdot M_{\mathcal{D}} \cdot M_{\breve{\mathcal{D}}}$ \\
Eq.(7) & $m+1$ & $2 M_{\mathcal{X}} \cdot M_{\mathcal{D}}$ \\
Eq.(8) & $m+1$ & $2 M_{\mathcal{y}} \cdot M_{\mathcal{D}}$ \\
\hline
\end{tabular}

Table 2. Free variables of the optimization task and the number of (scalar) symbolic decision variables they introduce into the optimization task.

\begin{tabular}{|c|c|c|}
\hline \multicolumn{2}{|c|}{$\begin{array}{c}\text { Matrix } \\
\text { variable }\end{array}$} & $\begin{array}{l}\text { number of (scalar) independent decision } \\
\text { variables appearing in the SDP }\end{array}$ \\
\hline \multicolumn{2}{|c|}{$\alpha$} & 1 \\
\hline \multicolumn{3}{|c|}{$P \quad 1 / 2 m(m+1)$} \\
\hline \multicolumn{3}{|c|}{$L_{\mathrm{b}} \quad m q$} \\
\hline & $\left(n+2 p+n^{2}+n p\right)\left(2 q+n^{2}+n q\right)$ \\
\hline \multicolumn{3}{|c|}{$L_{\mathrm{C}_{\mathrm{k}}}$ and $\bar{L}_{\mathrm{C}_{1}} \quad\left(M_{X}+M_{y}\right) \times m q$} \\
\hline \multicolumn{3}{|c|}{$M_{\mathrm{C}_{\mathrm{k}}}$ and $\bar{M}_{\mathrm{C}_{\mathrm{l}}} \quad\left(M_{X}+M_{\mathcal{Y}}\right) \times(m+1) n$} \\
\hline \multicolumn{3}{|c|}{ The dimensional parameters are the following: } \\
\hline \multicolumn{3}{|c|}{$P \quad$ number of elements in $\pi$} \\
\hline \multicolumn{3}{|c|}{$m \quad$ number of elements in $\pi_{\mathrm{b}}(n+p)$} \\
\hline \multicolumn{3}{|c|}{$q \quad$ number of rows in annihilator $\mathcal{N}_{\pi_{\mathrm{b}}}(x, \delta)$} \\
\hline \multicolumn{3}{|c|}{$\begin{array}{ll}M_{X} & \text { number of corner points of } \mathcal{X} \\
M_{\mathcal{Y}} & \text { number of corner points of } \mathcal{Y}\end{array}$} \\
\hline \multirow{2}{*}{\multicolumn{3}{|c|}{$\begin{array}{ll}M_{\mathcal{D}} & \text { number of corner points of } \mathcal{D} \\
M_{\breve{ }} & \text { number of corner points of } \widetilde{\mathcal{D}}\end{array}$}} \\
\hline & \multicolumn{2}{|c|}{$M_{\breve{\mathcal{D}}} \quad$ number of corner points of $\breve{\mathcal{D}}$} \\
\hline \multicolumn{3}{|c|}{$Q_{\mathrm{k}}^{\mathrm{T}} P_{\mathrm{C}_{\mathrm{k}}}^{(\alpha)}(x, \delta) Q_{\mathrm{k}} \geq 0 \quad \forall(x, \delta) \in \vartheta\left(\mathcal{F}_{\mathrm{k}}^{x} \times \mathcal{D}\right)$} \\
\hline \multicolumn{3}{|c|}{$\bar{Q}_{\mathrm{k}}^{\mathrm{T}} \bar{P}_{\mathrm{C}_{\mathrm{k}}}^{(1)}(x, \delta) \bar{Q}_{\mathrm{k}} \leq 0 \quad \forall(x, \delta) \in \vartheta\left(\mathcal{F}_{\mathrm{k}}^{\mathcal{y}} \times \mathcal{D}\right)$} \\
\hline
\end{tabular}

then $\varepsilon_{\alpha}$ is inside $\mathcal{X}$ and $\varepsilon_{1}$ is around $\mathcal{Y}$. Variables $Q_{\mathrm{k}}, \bar{Q}_{\mathrm{k}}$, $\bar{P}_{\mathrm{C}_{\mathrm{k}}}^{(1)}$, and $P_{\mathrm{C}_{\mathrm{k}}}^{(\alpha)}$ are defined in Eqs.(3.34) and (3.36) of Ref. [15], $\mathcal{F}_{\mathrm{k}}^{\mathcal{X}}$ denotes the $k^{\text {th }}$ facet of $\mathcal{X}$, furthermore, $M_{\mathcal{X}}$ denotes the number of facets of $\mathcal{X}$.

The LMI conditions of Eqs.(5)-(8) are affine parameter-dependent LMIs, which can be computationally handled by checking their feasibility at the corner points of the polytopic region, on which the parameters $(x, \delta, \dot{\delta})$ are defined. Depending on the number of the corner points, a given number of parameter independent LMI conditions (Table 1) is obtained.

The SDP task can be summarized as follows. In order to find the maximal invariant level set $\varepsilon_{\alpha}$ of the Lyapunov function, one should maximize $\alpha$, under the following conditions:

$$
\begin{array}{ll}
\text { Eq.(5) } & V(x, \delta) \text { is positive on } X \times \mathcal{D} \\
\text { Eq.(6) } & \dot{V}(x, \delta, \dot{\delta}) \text { is negative on } \chi \times \mathcal{D} \times \breve{\mathcal{D}} \\
\text { Eq.(7) } & \varepsilon_{\alpha} \text { lies inside } \chi \\
\text { Eq.(8) } & \mathcal{Y} \text { is inside } \varepsilon_{1} \\
\text { The number of LMIs and their dimensions are given }
\end{array}
$$
in Table 1, furthermore, the free variables of the optimization task are listed in Table 2. 


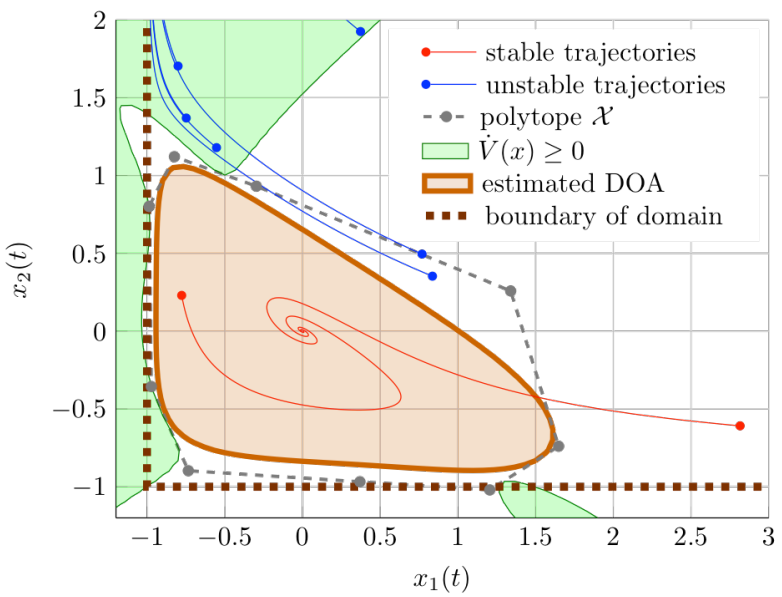

Figure 3. Phase diagram of the 2D locally stable system. Red trajectories converge at the origin, the blue ones do not converge at the origin. The estimated DOA and the used polytope $\mathcal{X}$ are illustrated by the orange-filled region and by the grey-dashed polytope, respectively. The green area highlights the subset of the state space where the Lyapunov function features a positive time-derivative.

\subsection{Finding the Most Appropriate Outer Polytope}

In the case of $2 \mathrm{D}$ systems, polytope $\mathcal{X}$ is evaluated manually through iterations. When having a higherdimensional system $(n \geq 3)$, the iterative procedure [6] is applied that starts from an initial polytope $\mathcal{X}^{(0)}$, then a new polytope $\chi^{(1)}$ is defined by enlarging the axis aligned bounding box of the obtained $\alpha$-level set $\varepsilon_{\alpha}^{(0)}$. During the iterations, $\chi^{(\mathrm{i})}$ is constrained to be a rectangular polytope. Fig.2 illustrates the operation of a single iteration step.

\section{Lotka-Volterra Systems}

The $N$-dimensional LV equation has the form

$$
\dot{\bar{x}}=\operatorname{diag}(\bar{x}+b), \quad A \in \mathbb{R}^{\mathrm{n} \times \mathrm{n}}, b \in \mathbb{R}^{\mathrm{n}},
$$

where $\operatorname{diag}(a), a \in \mathbb{R}^{\mathrm{n}}$ denotes an $n \times n$ square diagonal matrix with the $x_{1}, \ldots, x_{\mathrm{n}}$ on the main diagonal. The system is translated into its, by assumption, unique interior equilibrium point $x^{*}=-A^{-1} b$ by introducing the centred state vector $x=\bar{x}-x^{*}$. Then, an autonomous nonlinear system of the form $\dot{x}=\mathcal{A}(x) x$ is obtained, where the matrix function $\mathcal{A}$ can be expressed as: $\mathcal{A}(x)=\operatorname{diag}\left(x+x^{*}\right) A$. By applying the LFT and the further algebraic model transformation steps, a model is obtained in the representation of Eq.(4), where the entries of $\pi(x)$ are second order monomials of the state variables.

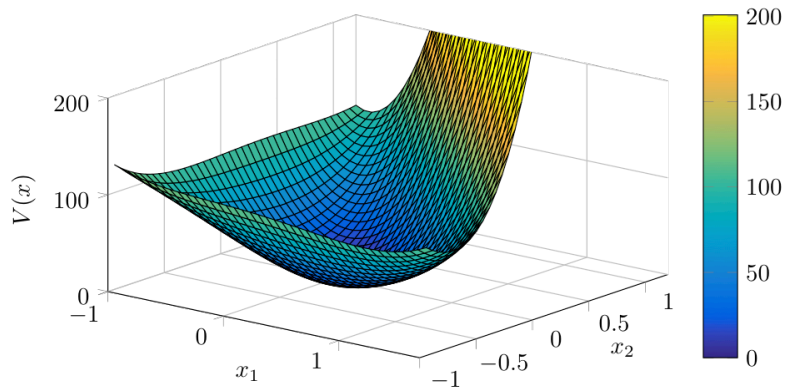

Figure 4. The obtained Lyapunov function in the case of the 2D LV system.

\section{Numerical Results}

In this section, the applicability of the approach presented above is illustrated through different locally stable LotkaVolterra models. The results presented here have been computed in a MATLAB environment. For symbolic computations, MATLAB'S built-in Symbolic Math Toolbox was used based on MuPAD. For linear fractional transformations (LFT), the Enhanced LFR-toolbox is used $[16,17]$. To model and solve semi-definite optimization (SDP) problems, Mosek solver with YALMIP was used [18].

\subsection{D Lotka-Volterra System}

A locally stable LV system is considered with the model matrix:

$$
A=\left[\begin{array}{cc}
-2 & -3 \\
1.4 & 1
\end{array}\right]
$$

with a unique interior equilibrium point $x^{*}=$ $\left[\begin{array}{ll}1 & 1\end{array}\right]^{\mathrm{T}}$. In Fig.3, some trajectories of the centred system can be observed from different initial conditions. The red trajectories converge at the equilibrium point, the blue ones do not tend to the equilibrium point. After solving the corresponding SDP, the obtained Lyapunov function is $V(x)=\pi_{\mathrm{b}}^{\mathrm{T}} P \pi_{\mathrm{b}}$ (Fig.4), where

$$
\begin{gathered}
\pi=\left[\begin{array}{lllll}
x_{1}^{2} & x_{1} x_{2} & x_{2}^{2}
\end{array}\right]^{\mathrm{T}}, \pi_{\mathrm{b}}=\left[\begin{array}{l}
x \\
\pi
\end{array}\right] \\
P=\left[\begin{array}{ccccc}
87.39 & 63.83 & -3.45 & 0 & 0 \\
63.83 & 165.26 & 67.24 & 100.85 & 28.76 \\
-3.45 & 67.24 & 7.63 & 0.15 & 0 \\
0 & 100.85 & 0.15 & 38.78 & 39.97 \\
0 & 28.76 & 0 & 39.97 & 15.53
\end{array}\right]
\end{gathered}
$$

In Fig.3, the shape of the manually chosen polytope $\mathcal{X}$, and the obtained invariant $\alpha$-level set of the Lyapunov function can be seen, which is considered as the estimated DOA (filled orange region). Areas can be observed, where the Lyapunov function's time derivative is positive (green region) can also be observed to be completely outside of $\mathcal{X}$. Fig.5 illustrates how the value of the Lyapunov function decreases along the trajectories. 
$\square \varepsilon_{\alpha}$, the invariant $\alpha$-level set of the Lyapunov function (estimated DOA)

$\because-x^{(i)}(t)$ : convergent trajectories, where the larger dots are the initial conditions, the smaller dots are the state values $x^{(i)}\left(t_{k}\right)$ at $t_{k} \simeq 1,2,3$

$v^{(i)}(t)=V\left(x^{(i)}(t)\right)$, values of the Lyapunov function along the trajectories
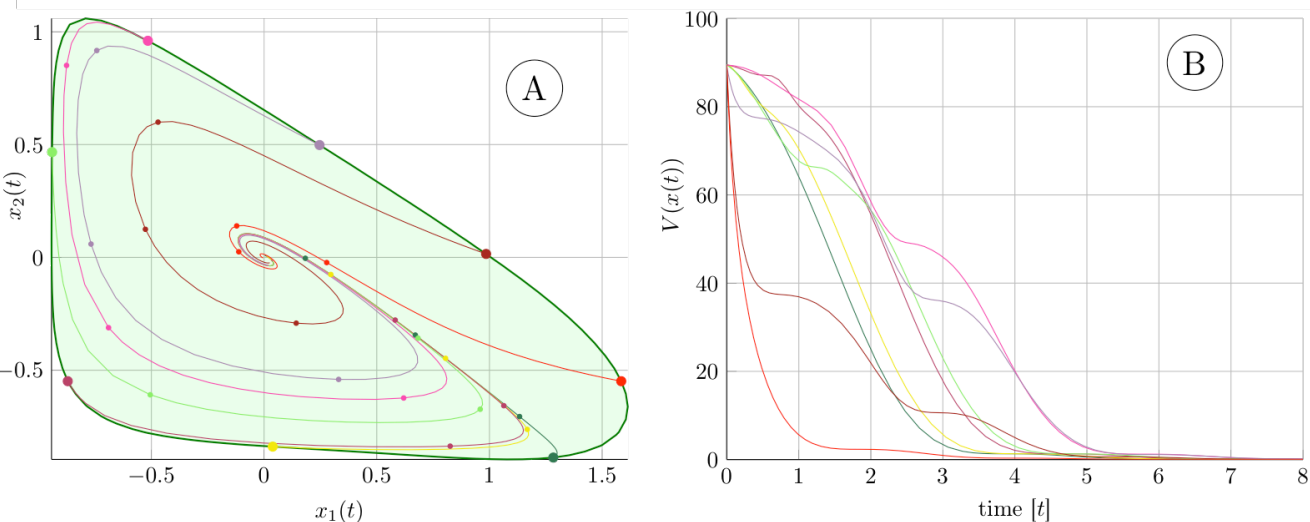

Figure 5. In Panel A, the obtained invariant level set is shown, furthermore some trajectories $x^{(\mathrm{i})}(t)$ of the system can be seen in different colors with initial conditions $x_{0}^{(\mathrm{i})}$ close to the boundary of the estimated DOA. The values of the Lyapunov function along the trajectories $\left(v^{(\mathrm{i})}(t)\right)$ are presented in Panel B, respectively.

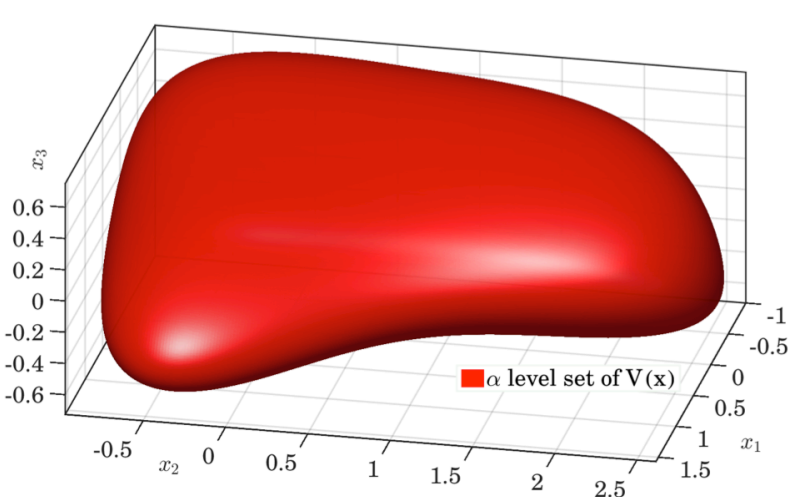

Figure 6. Estimated DOA of the locally stable 3D Lotka-Volterra system (red mesh) and the corresponding final polytope $\mathcal{X}$.

\subsection{D Lotka-Volterra System}

A locally stable 3D LV system has been chosen with the model matrix

$$
A=\left[\begin{array}{ccc}
0.06 & 0.21 & 0.83 \\
-2.47 & -2.10 & -3.64 \\
0.06 & 0.47 & -0.45
\end{array}\right]
$$

Similarly to the $2 \mathrm{D}$ example, the equilibrium point is set to $x^{*}=\left[\begin{array}{lll}1 & 1 & 1\end{array}\right]^{\mathrm{T}}$. The monomial set appearing in $\pi_{\mathrm{b}}$ contains the state variables and every possible $2^{\text {nd }}$ order monomial:

$$
\pi^{\mathrm{T}}=\left[\begin{array}{llllll}
x_{1}^{2} & x_{1} x_{2} & x_{1} x_{3} & x_{2}^{2} & x_{2} x_{3} & x_{3}^{2}
\end{array}\right]
$$

Matrix $P$ is a $9 \times 9$ symmetric matrix. Fig.6 illustrates the invariant $\alpha$-level set of the obtained Lyapunov function (3D red volume). In Fig.7, one can see the cross-sections of the 3D invariant region from three different viewpoints. Some trajectories of the system are shown in red (stable) and blue (unstable).
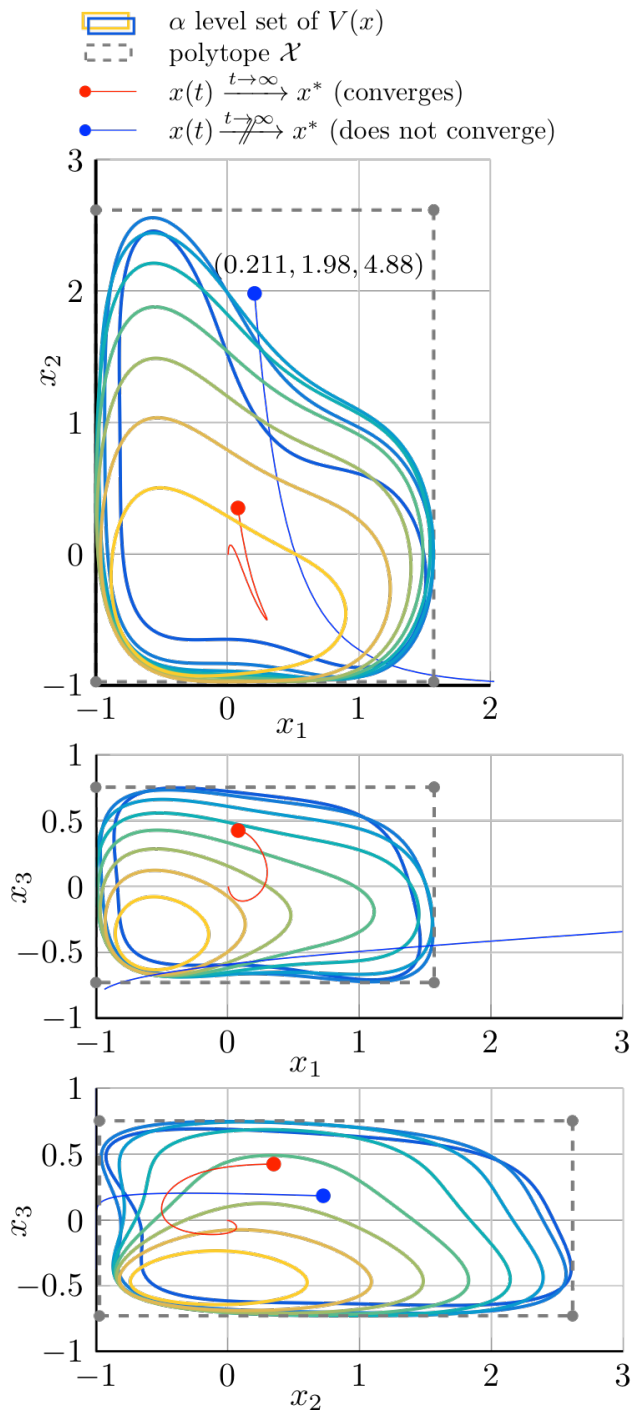

Figure 7. Cross-sectional view of the 3D estimate along the major planes $x_{\mathrm{i}}, x_{\mathrm{j}}$ and using different values for $x_{\mathrm{k}}$, where $(i, j, k)$ is a permutation of $(1,2,3)$. 
Table 3. Number and sizes of the LMIs in the case of $N$-dimensional LV systems.

\begin{tabular}{ccc}
\hline size & number & description \\
\hline$m_{\mathrm{b}} \times m_{\mathrm{b}}$ & $2^{\mathrm{n}}$ & positivity of $V(x)$ \\
$m_{\mathrm{a}} \times m_{\mathrm{a}}$ & $2^{\mathrm{n}}$ & negativity of $\dot{V}(x)$ \\
$m_{\mathrm{b}} \times m_{\mathrm{b}}$ & $n \cdot 2^{\mathrm{n}}$ & $\varepsilon_{\alpha} \in \mathfrak{I n t}(X)$ \\
$m_{\mathrm{b}} \times m_{\mathrm{b}}$ & $n \cdot 2^{\mathrm{n}}$ & $\mathcal{Y} \in \mathfrak{I n t}\left(\varepsilon_{\alpha}\right)$ \\
\hline
\end{tabular}

$m_{\mathrm{b}}$ is the size of the Lyapunov matrix $P, m_{\mathrm{a}}=1 / 2\left(n^{3}+5 n^{2}+4 n\right)$ is the size of the matrix $P_{\mathrm{a}}(P)$, which appears in the LMI ensuring the negativity of the time derivative of the Lyapunov function.

\subsection{D Lotka-Volterra System}

The method was applied to a locally stable 4D LV system with a unique interior equilibrium point:

$$
x^{*}=\left[\begin{array}{llll}
1 & 1 & 1 & 1
\end{array}\right]^{\mathrm{T}} .
$$

The model matrix of the LV system was chosen to be:

$$
A=\left[\begin{array}{llll}
-4.7126 & -1.5833 & 1.5346 & 2.3230 \\
-9.2461 & -3.1634 & 2.8648 & 2.7796 \\
-14.258 & -4.5477 & 3.6104 & 4.6238 \\
-3.1687 & -0.8016 & 1.2287 & 1.2656
\end{array}\right]
$$

Due to the fact that model matrix $A$ is a "full" matrix, i.e. there are no zero entries in it, every secondorder monomial will appear in the equation of the system. As a consequence, the LFT will produce a model Eq.(4), in which $\pi$ will contain every possible
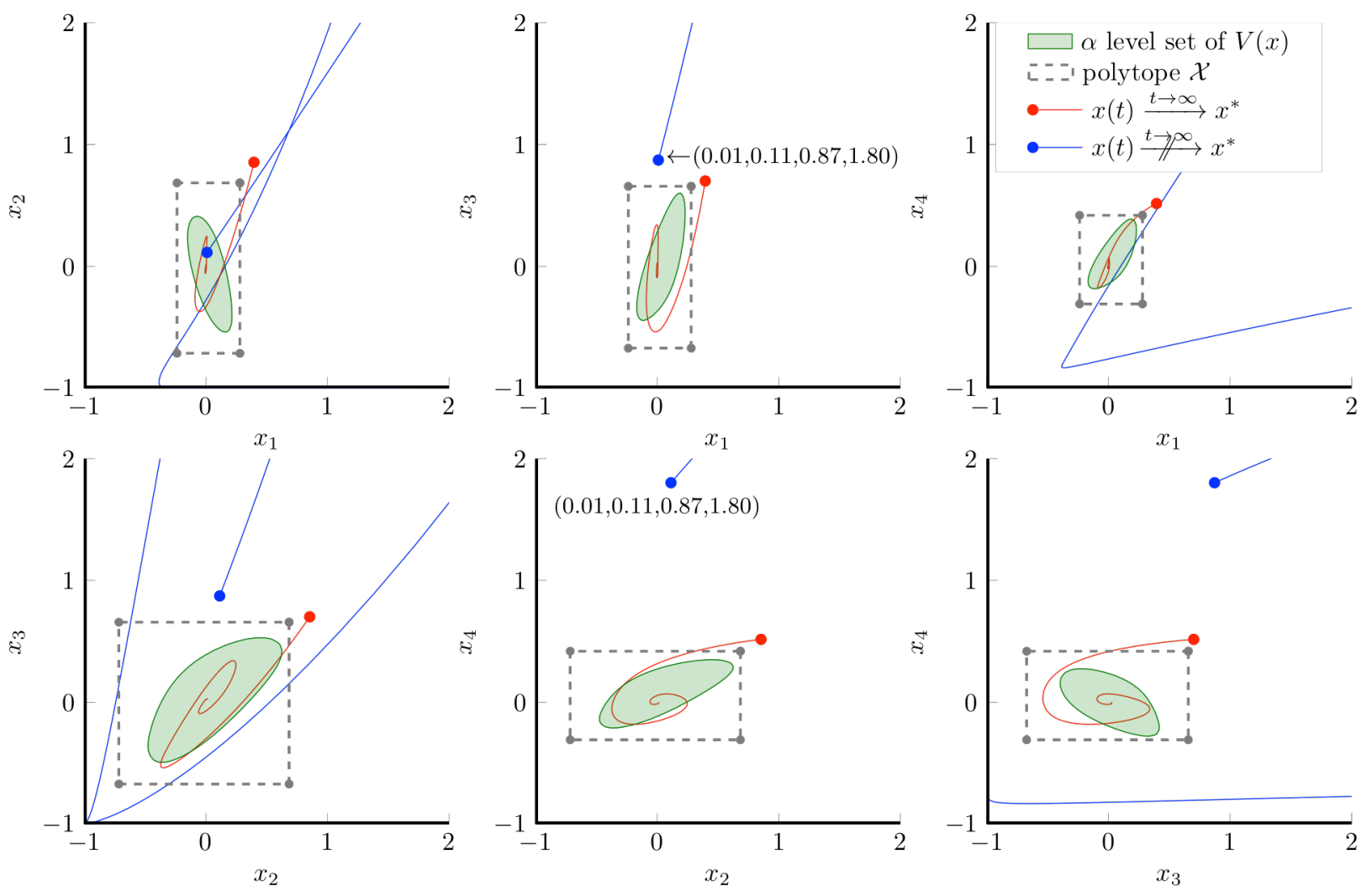
monomials (including the state variables) is $m_{\mathrm{b}}=\left(n^{2}+\right.$ $3 n) / 2$ in the case of a "full" model matrix.

In Table 3, the number and sizes of the LMIs have been summarised in the case of $N$-dimensional LV systems with a "full" model matrix. The exponential factor $2^{\mathrm{n}}$ in Table 3 originates from the rectangular shape of polytope $\mathcal{X}$ possessing $2^{\text {n }}$ corner points. On the other hand, the feasibility of LMI conditions in Eqs.(7) and (8) should be checked at each corner point of every facet of the polytope. Furthermore, an $N$-dimensional rectangular polytope has $2 n$ facets with $2^{\text {n-1 }}$ corner points. As can be seen, a rectangular polytope introduces an exponential increase in the dimension of the given problem. However, $\mathrm{N}$-dimensional intervals can be easily handled compared to arbitrarily shaped polytopes defined by (hyper)-triangle meshes. Fig.8 illustrates the cross sections of the invariant domain along the different pair of axes.

\subsection{D Uncertain LV System with an Uncertain Equilibrium Point}

In this section, the same 2-dimensional system is presented that appeared in Section 5.1 with the same model parameters but with an additional uncertain term $K \delta$, where $K=\left[\begin{array}{ll}1 & 1\end{array}\right]^{\mathrm{T}}$. It is assumes, that $\delta \in \mathcal{D}=$ $[-0.1,0.1]$ is an unknown constant parameter $(\dot{\delta}=0)$. The equation of the uncertain model is the following:

$$
\dot{\bar{x}}=\operatorname{diag}(\bar{x})(A \bar{x}+K \delta+b)
$$

second-order monomial. This means that the number of

Figure 8. Cross-sectional view of the DOA estimate of the 4D LV system. 
The equilibrium point of the uncertain system is an affine function of the uncertain parameter $\delta$ :

$$
x^{*}(\delta)=A^{-1}(-K \delta-\mathrm{b})=-A^{-1} K \delta+x_{0}^{*},
$$

where $x_{0}^{*}$ is the equilibrium point of the system presented in Section 5.1, i.e. when $\delta=0$. Eq.(15) is translated into the following form:

$$
\dot{\bar{x}}=\operatorname{diag}(\bar{x}) A\left(\bar{x}-x^{*}(\delta)\right) \text {. }
$$

The centred model of the system around the uncertain equilibrium point $x^{*}(\delta)$ can be calculated if the new state vector $x=\bar{x}-x^{*}(\delta)$ is introduced. The equation of the centred system is the following:

$$
\dot{x}=\operatorname{diag}\left(x+x^{*}(\delta)\right) A x .
$$

If the Lyapunov function depends on $\delta$, especially when possessing an uncertain equilibrium point, it is not straightforward to determine an invariant region by considering the $\alpha$-level set of the Lyapunov function. However, it is possible to compute a region $\mathfrak{J}$, which is "invariant with respect to" a larger region $\mathfrak{U}(\mathfrak{J} \subset \mathfrak{U})$, in the sense that every trajectory with an initial condition from $\mathfrak{J}$ will not leave $\mathfrak{U}$.

In order to compute $\mathfrak{I}$ and $\mathfrak{U}$, the $\alpha$-level set $\bar{\varepsilon}_{\alpha}(\delta)$ of the obtained Lyapunov function $V(x, \delta)$ is determined in the coordinates system of the original system and at the corresponding equilibrium point for every $\delta \in \mathcal{D}$. The overlining in the notation $\bar{\varepsilon}_{\alpha}(\delta)$ means that this level set is translated into the original coordinates system. As Fig.9 illustrates, the intersection $\mathfrak{J}$ and the union $\mathfrak{U}$ of the $\alpha$-level sets obtained for different values of $\delta$ have been computed. It can be stated that every trajectory starting from region $\mathfrak{I}$ will not leave region $\mathfrak{u}$.

\section{Conclusion}

In this work, the Lyapunov function of $N$-dimensional LV systems $(N=2,3,4)$ was successfully computed by using the improved optimization-based method [6, 15]. In the case of each deterministic system, an invariant region was given, as the estimated DOA. In the case of an uncertain LV system with an uncertain interior equilibrium point, two regions $(\mathfrak{J} \subset \mathfrak{U})$ were given, which describe the stable regions of the system.

\section{SYMBOLS}

$$
\begin{array}{ll}
\dot{x} & \text { denotes the time-derivative of function } x(t) \\
\mathbf{L}^{\mathrm{T}} & \text { denotes the transpose of a matrix } \mathbf{L} \\
\vartheta(\cdot) & \text { denotes the corner points of a polytope } \\
\operatorname{diag}(\mathbf{x}) & \text { denotes the diagonal matrix } \\
x=x(t) \in \mathbb{R}^{\mathrm{n}} \text { represents the state variables of a } \\
\text { dynamical system }
\end{array}
$$

$\bar{\varepsilon}_{\alpha}\left(\delta_{i}\right): \alpha$-level set of $V(x, \delta)$ for different values of $\delta$ in the original coordinates system

$\bullet \bar{x}^{*}\left(\delta_{i}\right)$ : equilibrium point for different values of $\delta$ I: intersection of $\bar{\varepsilon}_{\alpha}(\delta) \forall \delta \in \mathcal{D}$

$\mathfrak{U}$ : union of $\bar{\varepsilon}_{\alpha}(\delta) \forall \delta \in \mathcal{D}$

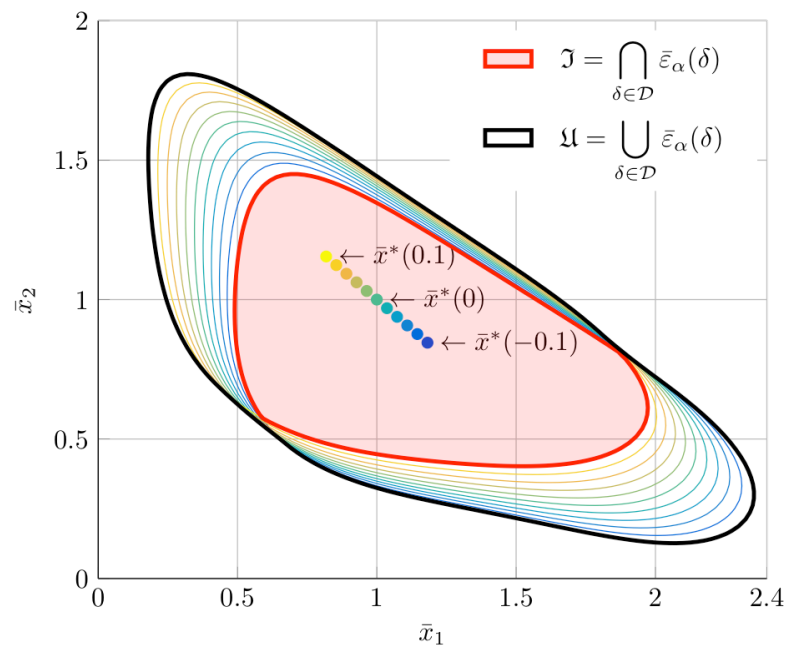

Figure 9. $\alpha$-level set $\varepsilon_{\alpha}\left(\delta_{\mathrm{i}}\right)$ of the Lyapunov function in the original coordinates system for some values $\delta_{i}$ of the uncertain parameter and the corresponding equilibrium point $x^{*}\left(\delta_{\mathrm{i}}\right)$ of the same color. The redfilled region is the intersection of the level sets for every possible value of $\delta$. The black line shows the contour of the union of the level sets.

$\delta \in \mathbb{R}^{\mathrm{n}} \quad$ vector of uncertain parameters

$\pi \in \mathbb{R}^{\mathrm{n}} \quad$ vector valued function, which represents the set of monomial and rational nonlinear terms to be considered in the Lyapunov function

$\pi \in \mathbb{R}^{\mathrm{m}=\mathrm{n}+\mathrm{p}}$ auxiliary variable denoting $\left[\begin{array}{l}x \\ \pi\end{array}\right]$

$V(x) \quad$ denotes the Lyapunov function

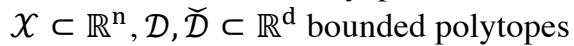

\section{Acknowledgement}

This research was supported by OTKA NF 104706

\section{REFERENCES}

[1] Chesi, G.: Domain of Attraction: Analysis and control via SOS programming (Springer-Verlag, London, UK) Vol. 415, 2011 DOI: 10.1007/978-085729-959-8

[2] Boyd, S.; El Ghaoui, L.; Feron, E., Balakrishnan, $\mathrm{V}$.: Linear matrix inequalities in system and control theory, in Studies in Applied Mathematics (SIAM, Philadelphia, PA, USA) Vol. 15, 1994 DOI: 10.1137/1.9781611970777

[3] Scherer, C.; Weiland, S.: Linear matrix inequalities in control, Lecture Notes (Dutch Institute for Systems and Control, Delft, The Netherlands) 2000 DOI: 10.1201/b10384-28

[4] Trofino, A.; Dezuo, T.J.M.: LMI stability conditions for uncertain rational nonlinear systems, Int. J. Robust Nonlinear Control 2013 24(18), 3124-3169 DOI: 10.1002/rnc.3047 
[5] Polcz, P.; Szederkényi, G.; Péni, T.: An improved method for estimating the domain of attraction of nonlinear systems containing rational functions, $J$. Physics: Conf. Ser. 2015 659(1), 012038 DOI: 10.1088/1742-6596/659/1/012038

[6] Polcz, P.; Szederkényi, G.; Hangos, K.M.: Computational stability analysis of an uncertain bioreactor model, Proc. Int. Symp. Stability, Vibration, and Control of Machines and Structures (SVCS 2016, Budapest, Hungary) pp. 1-12, 2016

[7] Rocha Filho, T.M.; Gléria, I.M.; Figueiredo, A.; Brenig, L.: The Lotka-Volterra canonical format, Ecol. Mod. 2005 183(1), 95-106 DOI: 10.1016/j.ecolmodel.2004.07.023

[8] Noonburg, V.W.: A neural network modeled by an adaptive Lotka-Volterra system, SIAM J. Appl. Math. 1989 49(6), 1779-1792 DOI: 10.1137/0149109

[9] Bhargava, S.: Generalized Lotka-Volterra equations and the mechanism of technological substitution, Technol. Forecast. Soc. Change 1989 35(4), 319-326 DOI: 10.1016/0040-1625(89)90068-1

[10] Goodwin, R.M.: A growth cycle, in Essays in Economic Dynamics (Palgrave, Macmillan, UK) pp. 165-170, 1982 DOI: 10.1007/978-1-349-05504-3

[11] Huu, A.N.; Costa-Lima, B.: Orbits in a stochastic Goodwin-Lotka-Volterra model, J. Math. Anal. Appl. 2014 419(1), 48-67 DOI: 10.1016/j.jmaa.2014.04.035
[12] Hernández-Bermejo, B.; Fairén, V.: LotkaVolterra Representation of general nonlinear systems, Math. Biosci. 1997 140(1), 1-32 DOI: 10.1016/S0025-5564(96)00131-9

[13] Takeuchi, Y.: Global dynamical properties of Lotka-Volterra systems (World Scientific, Singapore) 1996 DOI: 10.1142/9789812830548

[14] Plank, M.: Hamiltonian structures for the $N$ dimensional Lotka-Volterra equations, J. Math. Phys. 1995 36(7), 3520-3534 DOI: 10.1063/1.530978

[15] Polcz, P.; Szederkényi, G.: An improved method for estimating the domain of attraction of uncertain rational systems using linear matrix inequality conditions and SDP optimization, Technical Report (Faculty of Inform. Techn. and Bionics, Pázmány Péter Catholic Univ., Budapest, Hungary), 2016 users.itk.ppke.hu/ polpe/techreport2016apr.pdf

[16] Hecker, S.; Varga, A.; Magni, J.F.: Enhanced LFRtoolbox for MATLAB, Proc. IEEE Int. Symp. Computer Aided Control Systems Design pp. 2529, 2004 DOI: 10.1109/CACSD.2004.1393845

[17] Magni, J.F.: Linear fractional representation toolbox (Ver. 2.0) for use with MATLAB, 2006 www.cert. fr/dcsd/idco/perso/ Magni

[18] Loefberg, J.: YALMIP: a toolbox for modeling and optimization in MATLAB, Proc. CACSD Conference (Taipei, Taiwan), 2012 users.isy.liu.se/ johanl/yalmip 